\title{
Dufour and radiation effect on MHD boundary layer flow past a wedge through porous medium with heat source and chemical reaction \\ ${ }^{1}$ R.Mohapatra, ${ }^{2}$ H.Pattanayak \\ R.Mohapatra ${ }^{1}$ Department of Mathematics, I.H.S.E,
}

Siksha 'O' Anusandhan University, Bhubaneswar, Khandagiri, India

H.Pattanayak, Department of Mathematics

\section{ABSTRACT}

Magnetohydrodynamics (MHD) boundary layer flow past a wedge with the influence of thermal radiation, heat generation and chemical reaction has been analysed in the present study. This model used for the momentum, temperature and concentration fields. The principal governing equations is based on the velocity $u_{w}(x)$ in a nanofluid and with a parallel free stream velocity $u_{e}(x)$ and surface temperature and concentration. The governing nonlinear boundary layer equations for momentum, thermal energy and concentration are transformed to a system of nonlinear ordinary coupled differential equations by using suitable similarity transformation with fitting boundary conditions. The transmuted model is shown to be controlled by a number of thermo-physical parameters, viz. the magnetic parameter, buoyancy parameter, radiation conduction parameter, heat generation parameter, Porosity parameter, Dufour number, Prandtl number, Lewis number, Brownian motion parameter, thermophoresis parameter, chemical reaction parameter and pressure gradient parameter. Numerical elucidations are obtained with the legendary Nactsheim-Swigert shooting technique together with Runge-Kutta six order iteration schemes.

KEYWORDS: Nanofluid dynamics; wedge; Magnetic field; Thermal radiation; Heat generation; Dufour effect.

\section{Council for Innovative Research}

Peer Review Research Publishing System

Journal: JOURNAL OF ADVANCES IN MATHEMATICS

Vol.11, No.4

www.cirjam.com, editorjam@gmail.com 


\section{INTRODUCTION}

It is now well accepted fact that the terms magnetohydrodynamics (MHD), thermal radiation and heat generation extensively appear in various engineering processes. MHD is significant in the control of boundary layer flow and metallurgical processes. Again the thermal radiation and heat generation possessions may arise in high temperature ingredients processing operations. Ingredients may be intelligently designed therefore with judicious implementation of radiative heating to produce the desired characteristics. This recurrently occurs in agriculture, engineering, plasma studies and petroleum industries.

\section{Nomenclature}

\begin{tabular}{|c|c|c|c|}
\hline$a, c, m$ & $\imath$ Positive Constants & $B_{0}$ & magnetic induction \\
\hline$C$ & nanoparticle concentration & $C_{p}$ & specific heat capacity \\
\hline \multirow[t]{2}{*}{$C_{w}$} & nanoparticle concentration at wedge & $C_{\infty}$ & ambient nanoparticle concentration \\
\hline & & \multicolumn{2}{|c|}{ as y tends to infinity } \\
\hline$D$ & \multicolumn{3}{|l|}{ coefficient of mass diffusivity } \\
\hline$D_{B}$ & Brownian diffusion coefficient & $D_{T}$ & thermophoresis diffusion coefficient \\
\hline$g$ & acceleration due to gravity & & Grashof number \\
\hline$G_{m}$ & Modified Grashof number & $k_{r}$ & rate of chemical reaction \\
\hline Kc & chemical reaction parameter & $L_{e}$ & Lews number \\
\hline$K c^{*}$ & dimensional reaction coefficient & $k$ & thermal conductivity \\
\hline$N_{t}$ & thermophoresis parameter & $N_{b}$ & Brownian motion parameter \\
\hline$M$ & magnetic parameter & $P$ & fluid pressure \\
\hline $\operatorname{Pr}$ & Prandtl number & $q_{w}$ & heat flux \\
\hline$R_{e}$ & local Reynolds number & $S$ & Source parameter \\
\hline$Q$ & heat source parameter & $Q_{0}$ & heat generation constant \\
\hline$T$ & fluid temperature & & \\
\hline$T_{\infty}$ & ambient temperature as $y$ tends to in & & \\
\hline$T_{w}$ & temperature at the & & \\
\hline$u, v$ & velocity component along $x$ and $y$ & re & \\
\hline$(x, y)$ & Co-ordinate & & \\
\hline
\end{tabular}

\section{Greek symbol:}

$\begin{array}{llll}\alpha & \text { thermal diffusivity } & f(\eta) & \text { dimensionless velocity } \\ \beta & \text { pressure gradient parameter } & \beta_{c} & \text { co-efficent of massl expanasion } \\ \beta_{T} \quad \text { co-efficent of thermal expansion } & \lambda & \text { constant moving parameter } \\ (\rho c)_{p} \text { effective heatt capacity of the nanofluid } & \\ (\rho c)_{f} \text { heat capacity of the fluid } & & \\ \eta \quad \text { similarity variable } & \theta & \text { dimensionless temperature }\end{array}$




\begin{tabular}{|llll}
$\phi$ & dimensionless concentration & $\rho$ & density of fluid \\
$\mu$ & dynamic viscosity & $v$ & kinematic viscosity \\
$\kappa$ & vortex viscosity & $\sigma$ & charge density \\
$\lambda_{T}$ & thermal convective parameter & $\lambda_{M}$ & mass convective parameter \\
$\omega$ & angular velocity & $\gamma$ & Chemical reaction parameter
\end{tabular}

$\psi(x, y)$ stream function

\section{Subscripts:}

w quantities at the wall

$\infty \quad$ quantities at the free stream

Numerous flow complications under different aspects have been considered by the several scholars. The problem of natural convection along a vertical isothermal or uniform flux plate is a classical problem. However, Copiello and Fabbri [1] studied the effect of viscous dissipation on the heat transfer in sinusoidal profile finned dissipaters. Ghiya and Kavitha[2] discussed about forced flow of a Newtonian fluid due to a vertical stretching sheet with viscous dissipation. The effect of radiation on convective heat transfer problems have been examined by a number of researchers using principally algebraic approximations for the radiative transfer simulation. Khan et al $[3,4]$ studied about Heat Generation, Thermal Radiation and Chemical Reaction Effects on MHD Mixed Convection Flow over an Unsteady Stretching Permeable Surface and MHD Boundary Layer Radiative, Heat Generating and Chemical Reacting Flow Past a Wedge Moving in a Nanofluid.

A nanofluid is a fluid containing nanometer-sized particles, called nanoparticles. These fluids are engineered colloidal suspensions of nanoparticles in a base fluid. The nanoparticles used in nanofluids are typically made of metals, oxides, carbides, or carbon nanotubes. Common base fluids include water, ethylene glycol and oil. In recent years studies on nanofluid heat and mass transfer boundary layer laminar flow have attracted considerable attention. Nanotechnology [517] has been broadly used in several industrial applications. Nanofluids demonstrate anomalously high thermal conductivity, significant change in properties such as viscosity and specific heat in comparison to the base fluid, features which have attracted many researchers to perform in engineering applications. Kim[18] analyzed the Convective Instability and Heat Transfer Characteristics of Nanofluids. Kang et al. [19] experimentally investigated on nanofluids include thermal conductivity. Jang and Choi [20] reconnoitered nanofluid thermal conductivity parameter effects.Nield and Kuznestov [21] and Kuznestov and Nield [22] considered laminar convective nanofluid boundary layer flow in a porous medium, with Brownian motion and thermophoresis particle deposition effects and simple boundary conditions. Khan and Pop $[23,24]$ studied boundary layer heat-mass transfer free convection flows also in porous media of a nanofluid past a stretched sheet. M. Wahiduzzaman[25] discussed about Viscous Dissipation and Radiation Effects on MHD Boundary Layer Flow of a Nanofluid Past a Rotating Stretching Sheet. Hamad and Pop [26] reported transient hydro magnetic free convection rotating flow of a nanofluid. Md. Shakhaoath Khan et al. [27] analyzed the boundary layer nanofluid flow with MHD radiative possessions. Khan and Pop [28] investigates boundary layer heat and mass transfer analysis past a wedge moving in a nanofluid. The prime objective of the present attempt is to extend the analysis of Khan and Pop [28].

This study finds the effect of thermal radiation, heat generation and chemical reaction on themagneto hydrodynamic convection flow past a wedge moving in a nanofluid. This study also emphasised that Brownian motion and thermophoresis are significant mechanisms in nanofluid performance. This study is encouraged by precise application in materials processing which combines photopyroelectric thermal radiation and magnetic fields simultaneously to modify nanofluid properties. Verification of computations is demonstrated by comparison with previously published literature of Shakhaoath [29]. The present study is applicable to the manufacturing of magnetic nanofluids and chemical engineering operations involving electro-conductive nano fluid suspensions. There are relatively few studies [30-37] also focused on the MHD, convection, radiative heat transfer, heat generation and nanofluid also addressed application for further research.

\section{METHODS: MATHEMATICAL MODEL}

Here we consider the two dimensional MHD laminar boundary layer heat and mass transfer flow past an impermeable stretching wedge with the influence of thermal radiation, heat generation and chemical reaction and moving with the velocity $u_{w}(x)$ in a nanofluid, and the free stream velocity is $u_{e}(x)$, where $x$ is the coordinate measured along the surface of the wedge. The pattern of the physical configuration and coordinate system are shown in Figure 1 by following Khan and Pop [28]. 


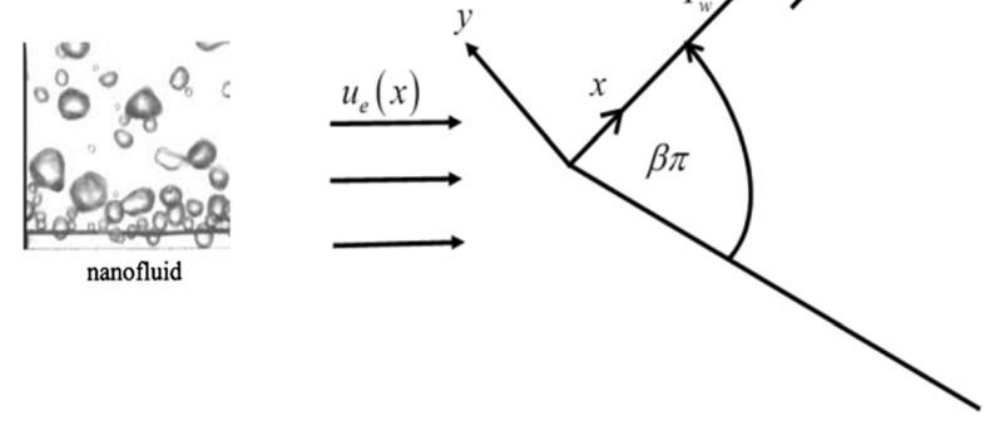

Fig.1 Physical configuration of a wedge

Here $u_{w}(x)>0$ corresponds to a stretching wedge surface velocity and $u_{w}(x)<0$ corresponds to a contracting wedge surface velocity, respectively. Instantaneously at time $t>0$, temperature of the plate and species concentration are raised to $T_{w}\left(>T_{\infty}\right)$ and $C_{w}\left(>C_{\infty}\right)$ respectively, which are thereafter maintained constant, where $T_{w}, C_{w}$ are temperature and species concentration at the wall and $T_{\infty}, C_{\infty}$ are temperature and species concentration far away from the plate respectively. A strong magnetic field $B=\left(0, B_{0}, 0\right)$ is applied in the y-direction. Under the above assumptions and usual boundary layer approximation, the MHD Mixed convective nanofluid flow governed by the following equations ([21] and [22]);

$$
\frac{\partial u}{\partial x}+\frac{\partial v}{\partial y}=0
$$

$u \frac{\partial u}{\partial x}+v \frac{\partial v}{\partial y}=u_{e} \frac{d u}{d x}+v \frac{\partial^{2} u}{\partial y^{2}}+g \beta_{T}\left(T-T_{\infty}\right)+g \beta_{C}\left(C-C_{\infty}\right)-\frac{\sigma B_{0}^{2} u}{\rho}-\frac{v u}{k^{\prime} p}$

$u \frac{\partial T}{\partial x}+v \frac{\partial T}{\partial y}=\alpha \frac{\partial^{2} T}{\partial y^{2}}++\frac{Q_{0}}{\rho C_{P}}\left(T-T_{\infty}\right)+\frac{16}{3} \frac{\sigma_{1} T_{\infty}^{3}}{\rho C_{P} k_{1}} \frac{\partial^{2} T}{\partial y^{2}}+\tau\left\{D_{B}\left(\frac{\partial T}{\partial y} \frac{\partial C}{\partial y}\right)+\frac{D_{T}}{T_{\infty}}\left(\frac{\partial T}{\partial y}\right)^{2}\right\}+\frac{D_{B}}{C_{\infty}} \frac{\partial^{2} C}{\partial y^{2}}$

$$
u \frac{\partial C}{\partial x}+v \frac{\partial C}{\partial y}=D_{B} \frac{\partial^{2} C}{\partial y^{2}}+\frac{D_{T}}{T_{\infty}} \frac{\partial^{2} T}{\partial y^{2}}-k_{r}\left(C-C_{\infty}\right)
$$

With the boundary conditions

$$
\left.\begin{array}{ll}
u=u_{w}(x)=-\lambda u_{e}(x), v=0, T=T_{w}, C=C_{w}, & \text { at } y=0 \\
u=u_{w}(x), \quad T \rightarrow T_{\infty}, C \rightarrow C_{\infty} & \text { as } y \rightarrow \infty
\end{array}\right\}
$$

In equation (2) the 3rd term on the right hand side is the convection due to thermal expansion and gravitational acceleration, the 4 th term on the right hand side is the convection due to mass expansion and gravitational acceleration and the 5 th term generated by the magnetic field strength because a strong magnetic field $B=\left(0, B_{0}, 0\right)$ is applied in the $y$-direction. Again in equation (3) the 2 nd term on the right hand side is the effect of heat generation on temperature flow and thermophoresis diffusion term due to nanofluid flow, 3rd term on the right hand side expressed the radiative [30] heat transfer flow, and is the rate of chemical reaction on the net mass flows, the last term indicates the Brownian motion due to nanofluid heat and mass transfer flow.

In order to conquers a similarity solution to equations (1) to (4) with the boundary conditions (5) the following similarity transformations, dimensionless variables are adopted in the rest of the analysis; 


$$
\left.\begin{array}{l}
\eta=y\left(\sqrt{\frac{(1+m) u_{e}}{2 x v}}\right), \psi=\sqrt{\left(\frac{2 u_{e} x v}{(1+m)}\right)} f(\eta), \theta=\theta(\eta)=\frac{T-T_{\infty}}{T_{w}-T_{\infty}} \\
\phi=\phi(\eta)=\frac{C-C_{\infty}}{C_{w}-C_{\infty}} \quad \text { and } \quad u=\frac{\partial \psi}{\partial y}, v=-\frac{\partial \psi}{\partial x}
\end{array}\right\}
$$

For the similarity solution of equations (1) to (4) with considering the value (from the properties of wedge,([28]) $u_{e}(x)=a x^{m}, u_{w}(x)=c x^{m} \quad,(0 \leq m \leq 1)$. Therefore, the constant moving parameter $\lambda$ is defined as $\lambda=c / a$, whereas $\lambda<0$ relates to a stretching wedge, $\lambda>0$ relates to a contracting wedge, and $\lambda=0$ corresponds to a fixed wedge, respectively. obtained as;

From the above transformations the non-dimensional, nonlinear, coupled ordinary differential equations are

$$
f^{\prime \prime \prime}(\eta)+f(\eta) f^{\prime \prime}(\eta)+\beta\left[1-f^{\prime}(\eta)^{2}\right]+\lambda_{T} \theta(\eta)+\lambda_{M} \phi(\eta)-(M+K p) f^{\prime}(\eta)=0
$$

$$
\left(\frac{1+R}{\operatorname{Pr}}\right) \theta^{\prime \prime}(\eta)+f(\eta) \theta^{\prime}(\eta)+N_{b} \theta^{\prime}(\eta) \phi^{\prime}(\eta)+N_{t} \theta^{\prime}(\eta)^{2}+Q \theta(\eta)+D u \phi^{\prime \prime}=0
$$

$\phi^{\prime \prime}(\eta)+\frac{N_{t}}{N_{b}} \theta^{\prime \prime}(\eta)+L_{e} f(\eta) \theta^{\prime}(\eta)-\gamma \operatorname{Re} L e \phi(\eta)=0$

The transformed boundary conditions are as follows;

$$
\left.\begin{array}{ll}
f=0, f^{\prime}=-\lambda, \theta=1, \phi=1 & \text { at } \eta=0 \\
f^{\prime}=1, \theta=0, \phi=0 & \text { as } \eta \rightarrow \infty
\end{array}\right\}
$$

where the notation primes denote differentiation with respect to $\eta$ and the parameters are defined as:

$$
\begin{aligned}
& M=\frac{\sigma B_{0}^{2} 2}{\rho(m+1) u_{e}}, \beta=\frac{2 m}{m+1}, G_{r}=\frac{2 g \beta_{T}\left(T_{w}-T_{\infty}\right)}{(m+1) v^{2}}, G_{m}=\frac{2 g \beta_{C}\left(C_{w}-C_{\infty}\right)}{(m+1) v^{2}}, \lambda_{T}=\frac{G_{r}}{\operatorname{Re}^{2}} \quad \lambda_{M}=\frac{G_{m}}{\operatorname{Re}^{2}}, \\
& \operatorname{Re}=\frac{x u_{e}}{v}, \operatorname{Pr}=\frac{v}{\alpha}, Q=\frac{2 Q_{0} x}{\rho C_{p}(m+1) u_{e}}, L_{e}=\frac{v}{D_{B}}, N_{b}=\frac{(\rho c)_{p} D_{B}\left(C_{w}-C_{\infty}\right)}{v(\rho c)_{f}} N_{t}=\frac{(\rho c)_{p} D_{T}\left(T_{w}-T_{\infty}\right)}{v T_{\infty}(\rho c)_{f}}, \\
& R=\frac{16}{3} \frac{\sigma_{1} T_{\infty}{ }^{3}}{\rho C_{p} k_{1} v}, \gamma=\frac{2 k_{1} v}{u_{e}^{2}(1+m)}, D u=\frac{D_{B}\left(C_{w}-C_{\infty}\right)}{C_{\infty} v\left(T_{w}-T_{\infty}\right)}, K p=\frac{2 v}{k p^{\prime}(1+m) u_{e}} x .
\end{aligned}
$$

\section{NUMERICAL (SHOOTING QUADRATURE) SIMULATIONS}

The non-dimensional, nonlinear, coupled ordinary differential equations (7) to (9) with boundary condition (10) are solved numerically using standard initially value solver the shooting method. For the purpose of this method, the NactsheimSwigert shooting iteration technique together with Runge-Kutta six order iteration scheme is taken which determines the temperature and concentration as a function of the coordinate $\eta$.

The boundary conditions equation (10) associated with the ordinary nonlinear differential equations of the boundary layer type is of the two-point asymptotic class. Two point boundary conditions have values of the independent variable specified at two different values of the independent variable. Specification of an asymptotic boundary condition implies the value of velocity approaches to unity and the value of temperature approaches to zero as the outer specified value of the independent variable is approached. The method of numerically integrating two-point asymptotic boundary value problem of the boundary layer type, the initial value method, requires that the problem be recast as an initial value problem. Thus it is necessary to set up as many boundary conditions at the surface as they are at infinity. The governing differential equations are then integrated with these assumed surface boundary conditions. If the required outer boundary condition is satisfied, a solution has been achieved. However, this is not generally the case. Hence a method must be devised to logically estimate the new surface boundary conditions for the next trial integration.

Asymptotic boundary value problems such as those governing the boundary layer equations are further complicated by the fact that the outer boundary condition is specified at infinity. In the trial integration infinity is numerically approximated by some large value of the independent variable. There is no a priori general method of estimating this value. Selection of too small a maximum value for the independent variable may not allow the solution to asymptotically converge to the required accuracy. Selecting a large value may result in divergence of the trial integration or in slow convergence of surface boundary conditions required satisfying the asymptotic outer boundary condition. Selecting too large a value of the 
independent variable is expensive in terms of computer time. Nachtsheim-Swigert developed an iteration method, which overcomes these difficulties. Extension of the iteration shell to above equation system of differential equations (10) is straightforward, there are three asymptotic boundary condition and hence three unknown surface conditions $f^{\prime \prime}(0), \theta^{\prime}(0)$ and $\phi^{\prime}(0)$.

\section{RESULTS AND DISCUSSION}

In order to get the physical insight to the system of ordinary differential equation (7)-(9) along boundary condition(10) are solved numerically by means of $4^{\text {th }}$ order R-K followed by shooting technique. The step size $\eta=0.05$ is used while obtaining the solution. The effects of pertinent physical parameter in the flow field are analyzed and discussed with the help of velocity, temperature, concentration profiles and tables.

Fig-2 reveals the effect of thermal Grashof Number $\left(\lambda_{T}\right)$ on the velocity profiles for different values of $k_{p}$ and $D u$. The thermal Grashof Number signifies the relative effects of the thermal buoyancy force to the viscous hydrodynamic force in the boundary layer. $\lambda_{T}>0$, is the positive values of $\lambda_{T}$ represents the cooling of the space. The present result is well agree with the result of Md Shakhaoath [29] by withdrawing the porous matrix $k_{p}$ and Dufour Number $D u$. It is also observed the velocity profile increases with the increases of $\lambda_{T}, k_{p}$ and $D u$. It is remarked that the profile has a pick near the plate and then decreases smoothly to the free steam velocity.

Fig-3 displays the effect of solutal Grashof Number $\left(\lambda_{m}\right)$ on the velocity profile for the both presence/absence of $k_{p}$ and $D u$. The solutal Grashof Number $\lambda_{m}$ defines the ratio of species buoyancy force to the viscous hydrodynamic force. It is observed that the velocity profile enhances with the increasing value of $\lambda_{m}$ for both increasing in $D u$ and the presence of porous matrix. The result is the good agreement with that of Shakhaoath [29] for the absence of $k_{p}\left(k_{p}=0\right)$ and $D u$ $(D u=0)$. Hence it is clear to note that both the buoyancy force have the same behavior on the velocity profile in the presence/absence of homogeneous/non-homogeneous Chemical reaction.

The effect of magnetic parameter for different values of porous matrix $\left(k_{p}\right)$ and Dufour Number $(D u)$ is shown in Fig-4. It is seen the increase velocity of $M$ leads to increase in velocity distribution. This is due to the fact of that applied transverse magnetic field produces a Lorenz force which beneficial to increase in profile of all points.

Fig-5 presents the variation of radiation parameter $R$ for different $k_{p}$ and $D u$ on velocity profile. From the figure it is cleared that the profile has a 2 layer characteristic for the absence of $R(R=0)$ and presence of $R(R=2)$ $\operatorname{Du}(D u=0) k_{p}\left(k_{p}=0\right)$ shown by dotted and bold lines respectively. It is observed this radiation parameters enhances the velocity profile in both the absence/ presence of $k_{p}$ and $D u$. It is further revealed that the absence of $k_{p}\left(k_{p}=0\right)$ and $D u(D u=0)$ is taken as a particular case of the previous author [29], resulting is a good agreement.

Fig-6 exhibits the effects of suction parameter $(\lambda)$ on the velocity profile in both the presence/absence of porous matrix keeping the other parameter is fixed. Suction acts to increase adherence of fluid to the wall which is terns referred the flow and hence decrease in the velocity profile of all points in the boundary layer, where as increase in porous matrix the velocity profile increases. Here it is concluded that suction has a retarding effect on the profile.

Fig-7 represents the effect of the thermal Grashof Number $\left(\lambda_{T}\right)$ of the temperature profile for different values of porous matrix $k_{p}$ and Dufour Number $D u$. The particular case of Shakhaoath [29] is also shown by withdrawing the porous matrix $\left(k_{p}=0\right)$ and Dufour number $(D u=0)$. The present result is in good agreement with the earlier published result. It is seen that the absence of both $k_{p}$ and $D u$ the thermal boundary layer becomes maximum. But the profile decreases with the increase of value $\lambda_{T}$. It is also remark that in the presence of with the absence of $D u(D u=0)$ the profile decreases in its boundary layer where as the presence of $D u$ enhance it.

The effect of magnetic parameter in the temperature profile is shown in Fig-8. From the profile it is clear that temperature decreases with the increase in magnetic parameter which produce a drag in the form of Larentz force in the presence of permeability parameter. Further temperature profile decreases in the presence of $k_{p}\left(k_{p}=1\right)$. But reverse effect enhanced in the presence of $D u$. 
Fig -9 exhibits the effect of radiation parameter on the temperature profile for the different values of $k_{p}$ and $D u$. It is clear to note that in the absence of $k_{p}=0, D u=0$ and in the absence of $R(R=0)$ the result is good agreement with that of Shakhaoath [29]. It is observed that presence of $k_{p}$ decreases the profile of all points in the boundary layer. Where in the presence of $D u$ enhance it. Further, increase in $R$ in the presence of $D u$ also decreases the temperature in the boundary layer.

The dimensionless temperature distribution for different values of thermophoresis parameter $N t$ and Brownian motion parameter $\mathrm{Nb}$ are illustrated in Fig-10. It interesting to note that increasing value of $N t$ and $N b$ increases the temperature profile significantly. The thermophoresis force generated by the temperature gradient creates a fast flow from the surface. In this way more fluid is heated away from the surface and consequently, as $N t$ increases, temperature increases in the boundary layer. Also both in increase in the Brownian motion parameter the random motion of particle increase with result in on enhancement in the temperature profile.

Fig-11 reveals the effect of $N t$ and $N b$ in the concentration profile. It is remarked that there is a sharp fall in concentration near plate, with in the domain $\eta \leq 0.5$ and this becomes smooth to meet the adequate boundary condition. It is seen that increase in $N t$ there is a decrease in concentration near the plate, but the increase in remarkable at the ambient state where in $\mathrm{Nb}$ decreases the concentration profiles.

Fig-12 exhibits the effect of chemical reaction parameter for different values of $k_{p}$ and $D u$. It is noted in $\gamma<0$ represents the exothermic chemical reaction, $\gamma=0$ represents no chemical reaction, $\gamma<1$ is an endothermic chemical reaction. The concentration profile decreases with the increase in $1^{\text {st }}$ order chemical reaction parameter for both presence/absence porous matrix $k_{p}$ and Dufour Number $D u$ respectively. It is also observed that increase in $k_{p}$ and

( $\lambda$ ) $D u$ decrease the concentration profile of all points of the boundary layer.

\section{REFERENCES}

[1] Copiello D. Fabbri G., 2008“Effect of viscous dissipation on the heat transfer in sinusoidal profile finned dissipaters," Heat Mass Trans. 44599-605

[2] Ghiya N. Kavitha T. 2013 Forced flow of a Newtonian fluid due to a vertical stretching sheet with viscous dissipation, International Journal of Engineering Science and Innovative Technology (IJESIT), 2(4), 132-143.

[3] Khan M.S., Karim I. and Biswas H.A. 2012 Heat Generation, Thermal Radiation and Chemical Reaction Effects on MHD Mixed Convection Flow over an Unsteady Stretching Permeable Surface. International Journal of Basic and Applied Science, 1, 363-377.

[4] Khan M.S. Karim, I., Islam, M.S. and Wahiduzzaman, M. MHD Boundary Layer Radiative, Heat Generating and Chemical Reacting Flow Past a Wedge Moving in a Nanofluid.Nano Convergence,1,20.

[5] M.M. Macdevette, T.G. Myers, B. Wetton, Boundary Layer Analysis And Heat Transfer OF A Nanofluid.

[6] Jang, S.P. and Choi, S.U.S.2007 Effects of Various Parameters on Nanofluid Thermal Conductivity. Journal of Heat Transfer, 129, 617-623.

[7] Khan W.A. and Pop I. 2010 Boundary-Layer Flow of a Nanofluid Past a Stretching Sheet. International Journal of Heat and Mass Transfer, 53, 2477-2483.

[8] Bég, O.A. Khan M.S., Karim I. Alam M.M. Ferdows M. 2013 Explicit Numerical Study of Unsteady Hydromagnetic Mixed Convective Nanofluid Flow from an Exponentially Stretching Sheet in Porous Media. AppliedNanoscience,4, 943957.

[9] Ferdows, M., Khan, M.S., Alam, M.M. Sun, S. 2012 MHD Mixed Convective Boundary Layer Flow of a Nanofluid through a Porous Medium Due to an Exponentially Stretching Sheet. Mathematical Problems in Engineering,2012.

[10] Ferdows M. Khan M.S., Beg O.A. Azad, M.A.K. Alam, M.M. 2013 Numerical Study of Transient Magnetohydrodynamic Radiative Free Convection Nanofluid Flow from a Stretching Permeable Surface. Journal of Process Mechanical Engineering, 228, 181-196.

[11] Khan, M.S., Alam, M.M. Ferdows, M.2011 Finite Difference Solution of MHD Radiative Boundary Layer Flow of a Nanofluid Past a Stretching Sheet. Proceeding of the International Conference of Mechanical Engineering 2011, 1-7.

[12] Khan, M.S., Alam, M.M. Ferdows, M. 2013 Effects of Magnetic Field on Radiative Flow of a Nanofluid Past a Stretching Sheet. Procedia Engineering,56,316-322.

[13] Khan, M.S., Karim, I., Ali, L.E. Islam, A. 2012 Unsteady MHD Free Convection Boundary-Layer Flow of a Nano-fluid along a Stretching Sheet with Thermal Radiation and Viscous Dissipation Effects. International NanoLetters,2,24.

[14] Khan, M.S., Karim, I. Biswas, H.A. 2012Non-Newtonian MHD Mixed Convective Power-Law Fluid Flow over a Vertical 
Stretching Sheet with Thermal Radiation, Heat Generation and Chemical Reaction Effects. Academic Research International, 3, 80-92.

[15] Khan, M.S., Karim, I. Islam, M.S.2014 MHD Buoyancy Flows of Cu, Al2O3 and TiO2 Nanofluid near Stagnation-Point on a Vertical Plate with Heat Generation. Physical SciencelnternationalJournal,4,754-767..

[16] Khan, M.S., Karim, I. Islam, M.S.2014 Possessions of Chemical Reaction on MHD Heat and Mass Transfer Nanofluid Flow on a Continuously Moving Surface. American Chemical Science Journal, 4, 401-415.

[17] Wahiduzzaman ,M.Khan and Karim I., MHD convective stagnation flow of nanofluid over a shrinking surface with thermal radiation, Heat Generation and Chemical Rection, Procedia Engineering, Accept,2015 .

[18] Kim, J.Kang, Y.T. Choi, C.K. 2004 Analysis of Convective Instability and Heat Transfer Characteristics of Nanofluids. Physics of Fluids, 16, 2395-2401.

[19] Kang HU, Kim SH. Oh JM.2006 Estimation of thermal conductivity of nanofluid using experimental effective particle. Exp. Heat Transfer 19, 181-191.

[20] Jang SP. Choi SUS.2007 Effects of various parameters on nanofluid thermal conductivity. ASME J. Heat Transfer $129,617-623$.

[21] Nield A. Kuznetsov AV. 2009 The Cheng-Minkowycz problem for natural convective boundary-layer flow in a porous medium saturated by a nanofluid. Int. J. Heat and Mass Transfer 52, 5792-5795 .

[22] Kuznetsov AV. Nield DA.2010 Natural convective boundary-layer flow of a nanofluid past a vertical plate. Int. J. of Thermal Sci. 49, 243-247.

[23] Khan WA, Pop I,2010 Boundary-layer flow of a nanofluid past a stretching sheet. Int. J. Heat and Mass Trans. 53, 2477-2483.

[24] WA Khan, I Pop, Free convection boundary layer flow past a horizontal flat plate embedded in a porous medium filled with a nanofluid. ASME J. Heat Transfer 133, 157-163 (2011)

[25] Wahiduzzaman M. , Khan Md. Shakhaoath, Biswas P. Karim Ifsana, Uddin M. S. 2015 Viscous Dissipation and Radiation Effects on MHD Boundary Layer Flow of a Nanofluid Past a Rotating Stretching Sheet, . Applied Mathematics, 6, 547-567

[26] Hamad MAA, Pop I,2012 Unsteady MHD free convection flow past a vertical permeable flat plate in a rotating frame of reference with constant heat surface in a nanofluid. Heat Mass Transfer 47, 1517-1524

[27] Khan MS, Alam MM. Ferdows M.2013 Effects of magnetic field on radiative flow of a nanofluid past a stretching sheet. Procedia Engineering Elsevier 56, 316-322

[28] Khan WA, Pop I,2013 Boundary layer flow past a wedge moving in a nanofluid. Math Probl. Eng., 1-7

[29] Khan Md Shakhaoath, Karim Ifsana, Islam Md Sirajul, Wahiduzzaman Mohammad.2014 MHD boundary layer radiative heat generating and chemical reacting flow past a wedge moving in a nanofluid, Nano Convergence,1-20

[30] Shakhaoath K Md, Ifsana K, Lasker Ershad A, Ariful I.2012 MHD free convection boundary layer unsteady flow of a nanofluid along a stretching sheet with thermal radiation and viscous dissipation effects. Int. Nano. Lett. 2(24), 1-9

[31] Khan MS, Wahiduzzaman M, Sazad MAK, Uddin MS.2012 Finite difference solution of MHD free convection heat and mass transfer flow of a nanofluid along a Stretching sheet with Heat generation effects. Indian J. Theor. Physics 60(4), 285-306

[32] Shakhaoath K Md, Ifsana K, Alam MM, Ferdowsand M, Beg OA,2013Explicit numerical study of unsteady hydromagnetic mixed convective nanofluid flow from an exponential stretching sheet in porous media. Appl. Nanosci. $18,1-15$

[33] Shakhaoath K Md, Ifsana K, Haider Ali 2012 Heat generation, thermal radiation and chemical reaction effects on MHD mixed convection flow over an unsteady stretching permeable surface. Int. J. Basic Appl. Sci. 1(2), 363-377.

[34] Shakhaoath K Md, Ifsana K, Haider Ali B Md,2012 Non-Newtonian MHD mixed convective power-Law fluid flow over a vertical stretching sheet with thermal radiation, heat generation and chemical reaction effects. Acad. Res. Int. 3(3), 8092

[35] Shakhaoath K Md, Ifsana K, Sirajul I Md, 2014 Possessions of chemical reaction on MHD heat and mass transfer nanofluid flow on a continuously moving surface. Am. Chem. Sci. J. 4(3), 401-415

[36] Khan WA, Gorla RSR,2012 Heat and mass transfer in power-law nanofluids over a non-isothermal stretching wall with convective boundary condition. ASME. J. Heat Transfer 134, 1-7.

[37] Mahmood M, Asghar S, Hossain MA 2009. ydromagnetic flow of viscous incompressible fluid past a wedge with permeable surface. J. Appl. Math Mechanics 89, 174-188.

\section{ACKNOWLEDGMENTS}

I sincerely convey my heartfelt -thanks to S.R. Mishra for his constant support. He has been a source of encouragement throughout the development of the paper. 


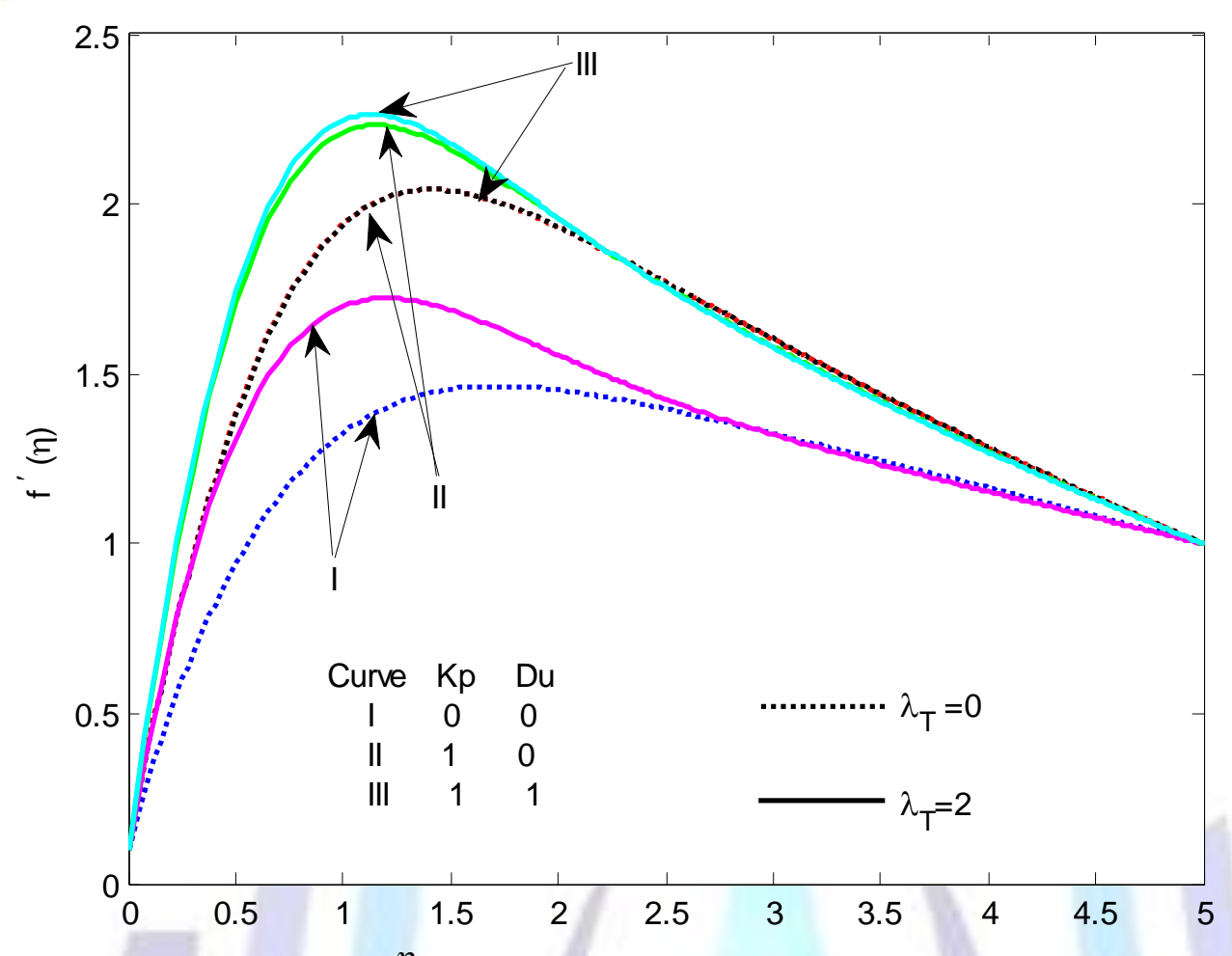

Fig. 2 Effect of $\lambda_{\mathrm{T}}, \mathrm{Kp}$ and Du on velocity profile

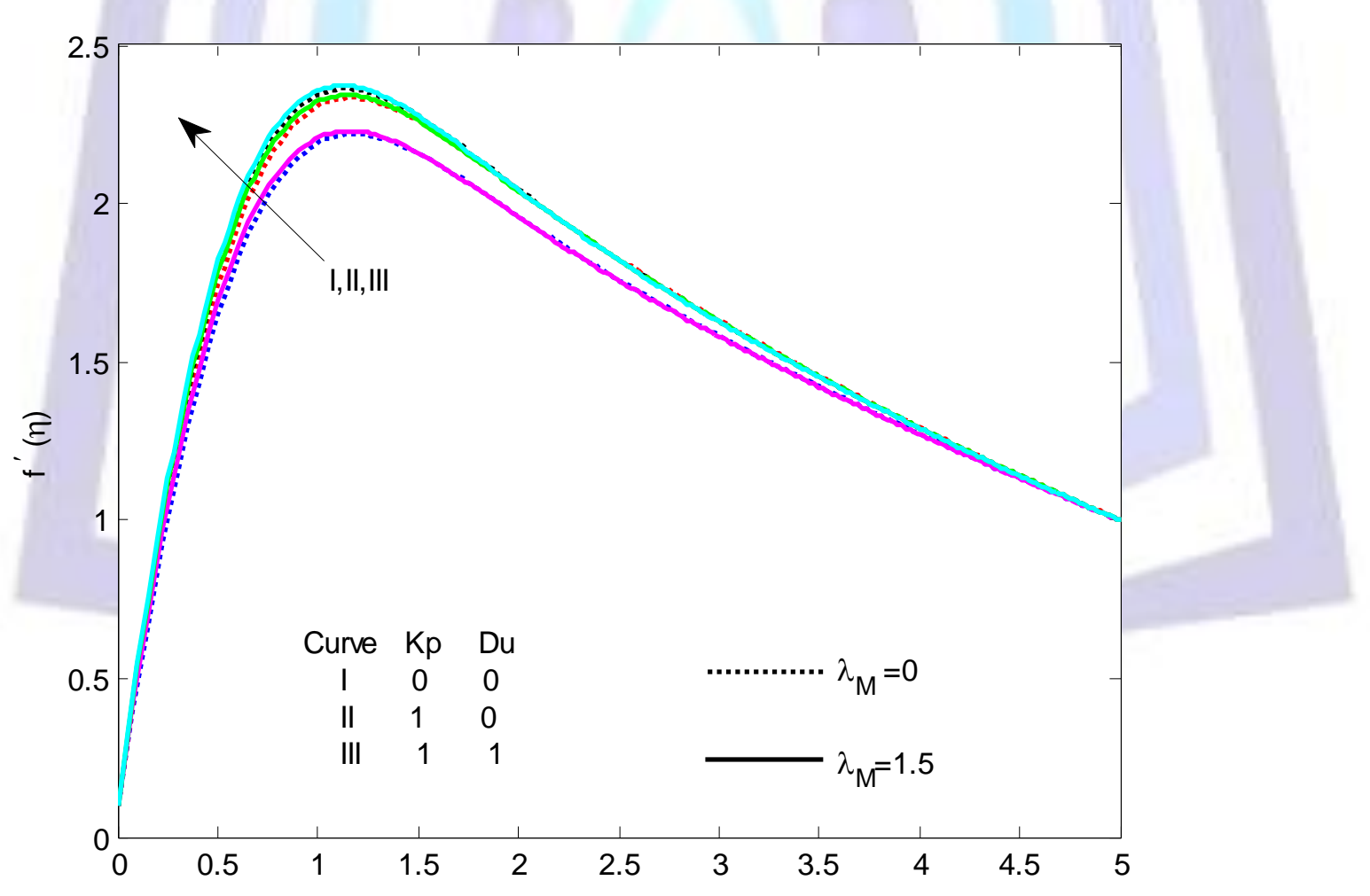

Fig.3 Effect of $\lambda_{\mathrm{M}}, \mathrm{Kp}$ and Du on velocity profile 


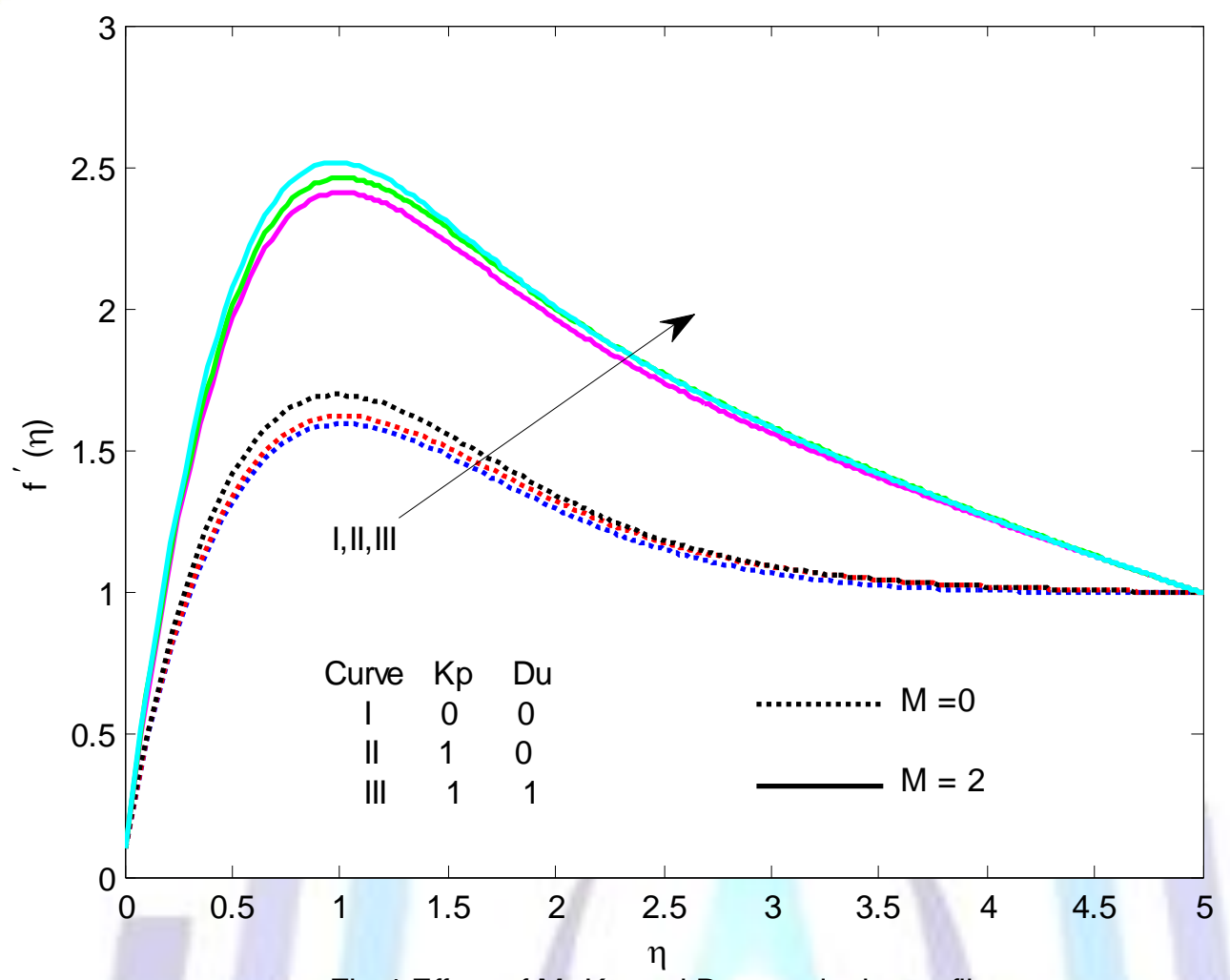

Fig.4 Effect of M, Kp and Du on velocity profile



Fig.5 Effect of $\mathrm{R}, \mathrm{Kp}$ and Du on velocity profile 

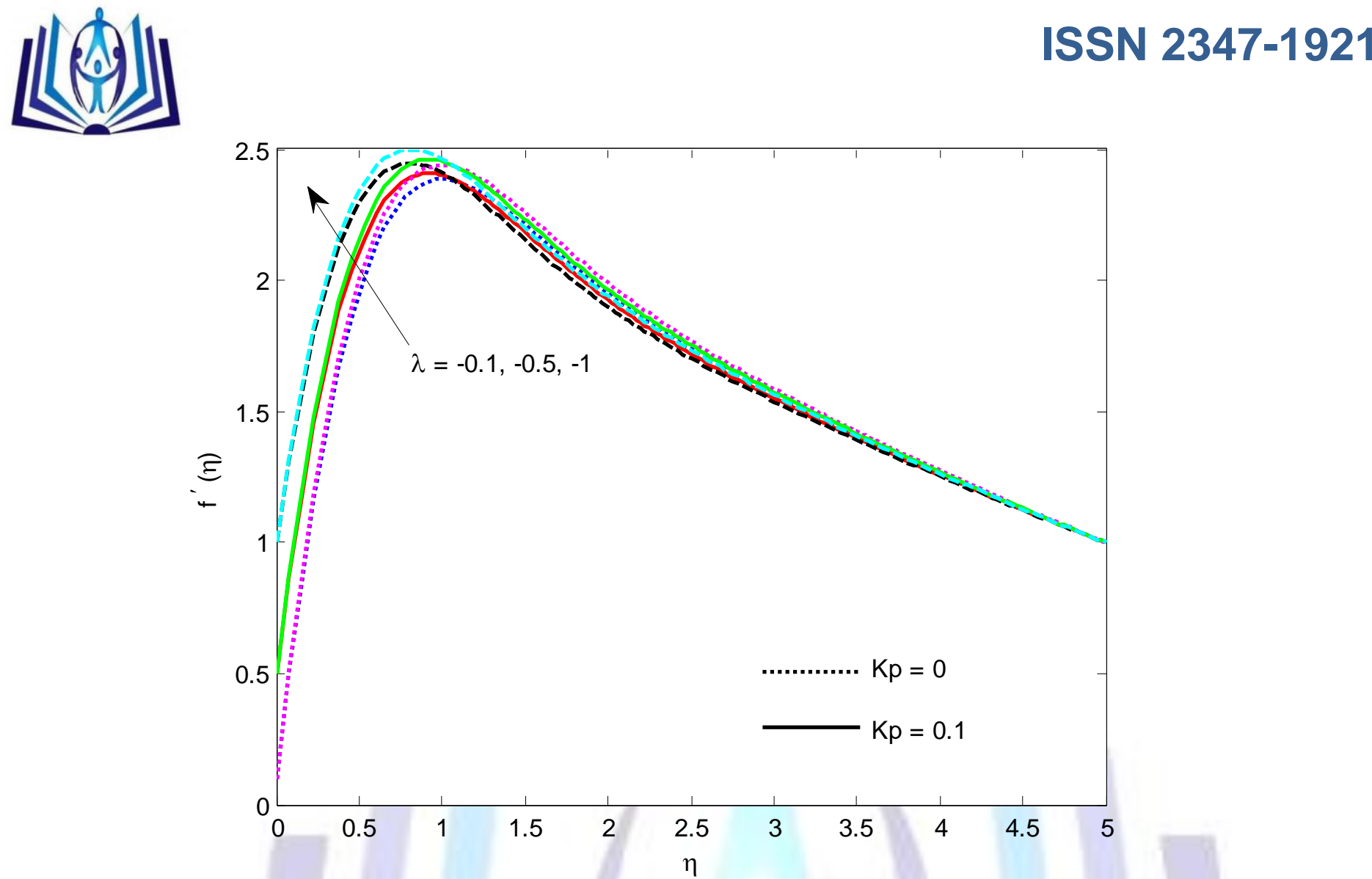

Fig.6 Effect of $\lambda$ and Kp on velocity profile

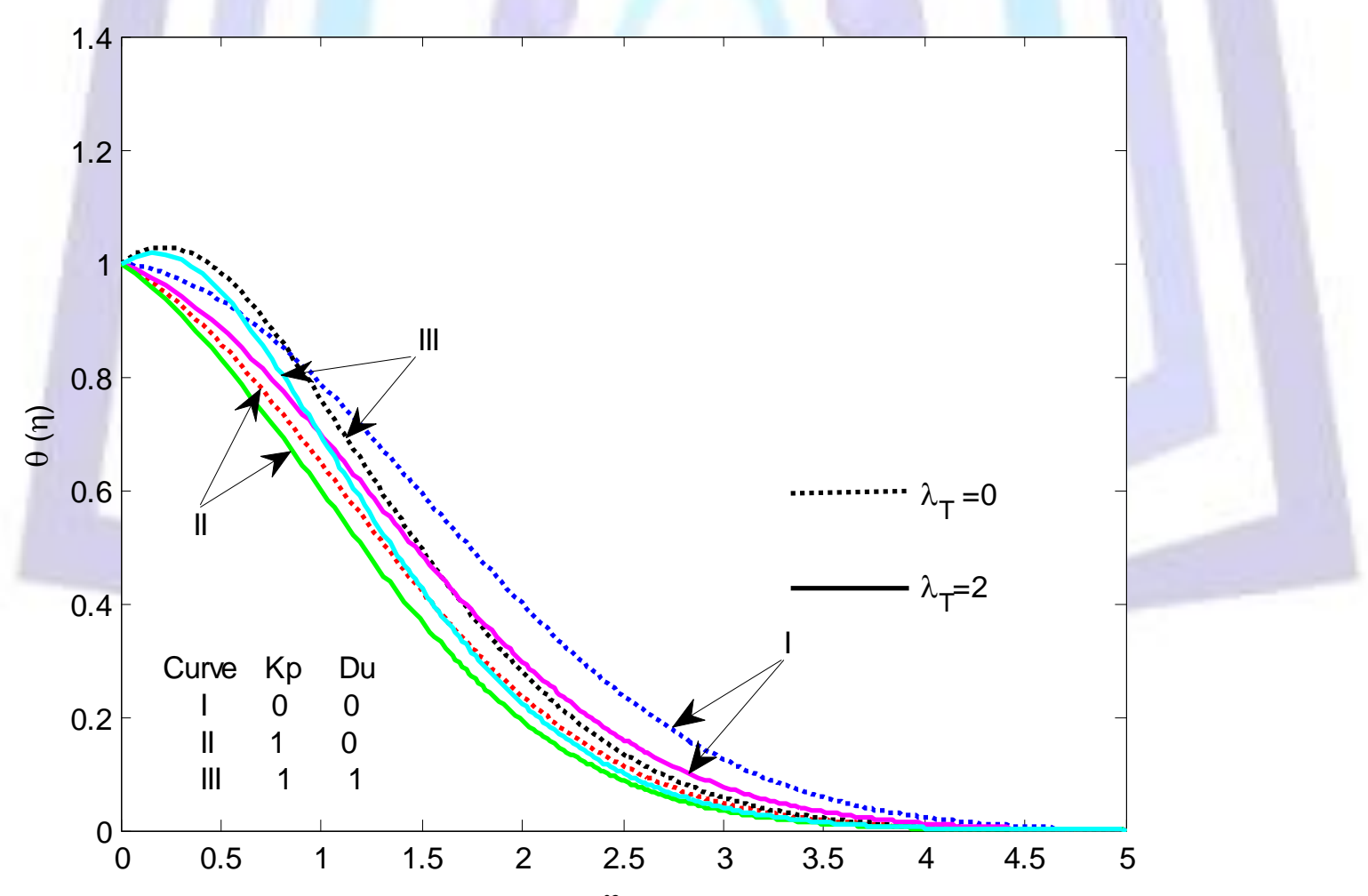

Fig.7 Effect of $\lambda_{\mathrm{T}}, \mathrm{Kp}$ and $\mathrm{Du}$ on Temperature profile 

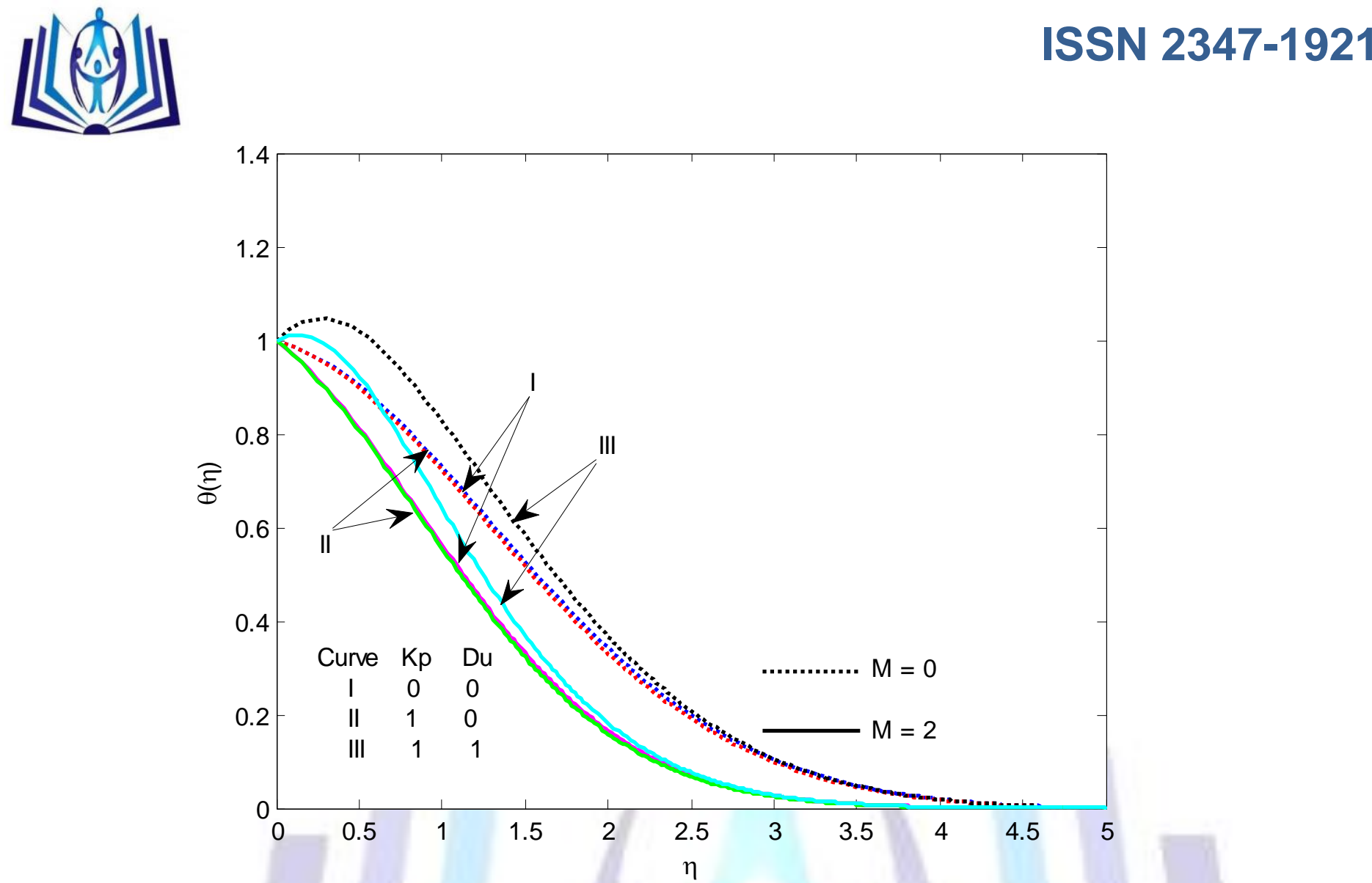

Fig.8 Effect of M, Kp and Du on temperature profile

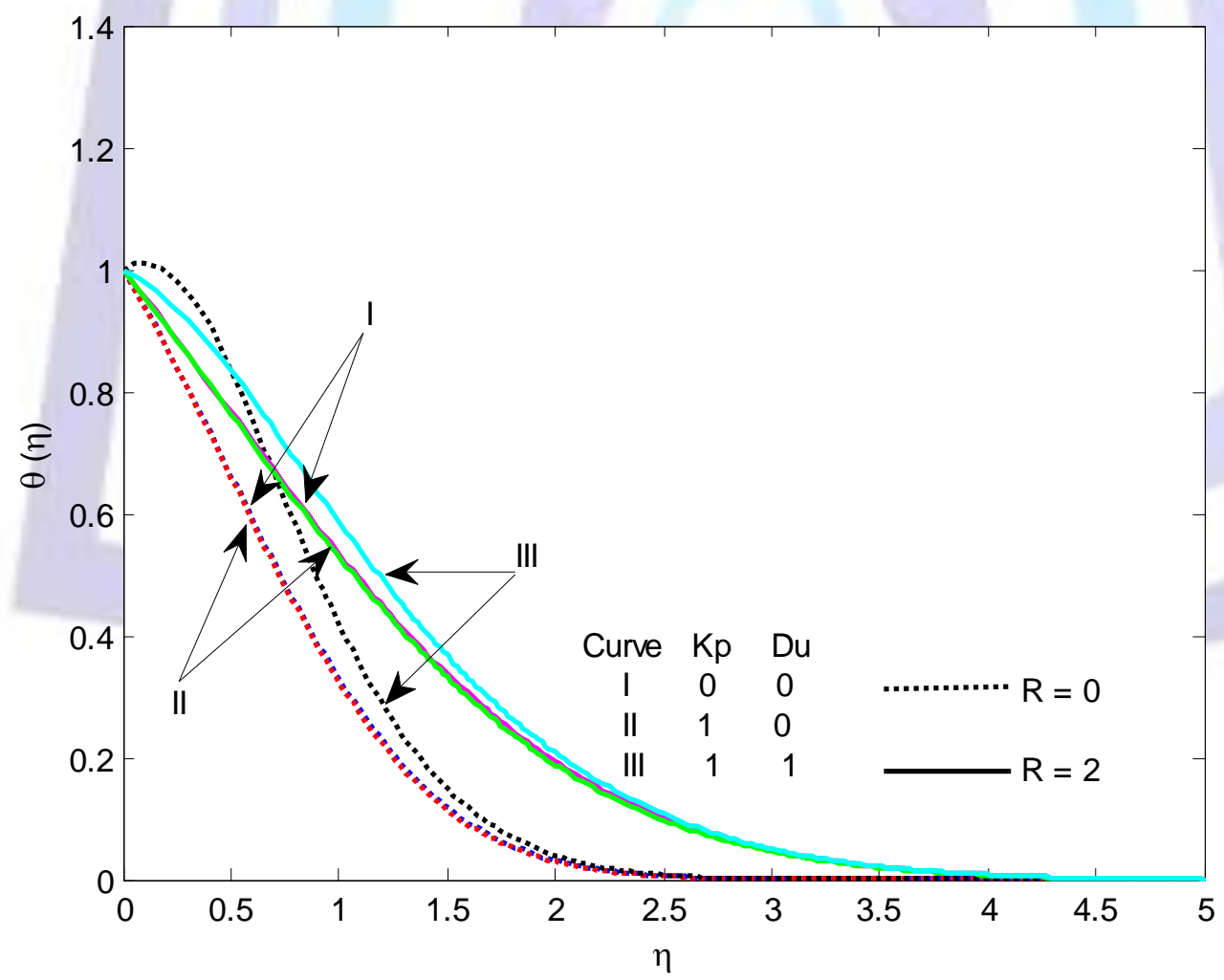

Fig.9 Effect of R Kp and Du on the temperature profile 


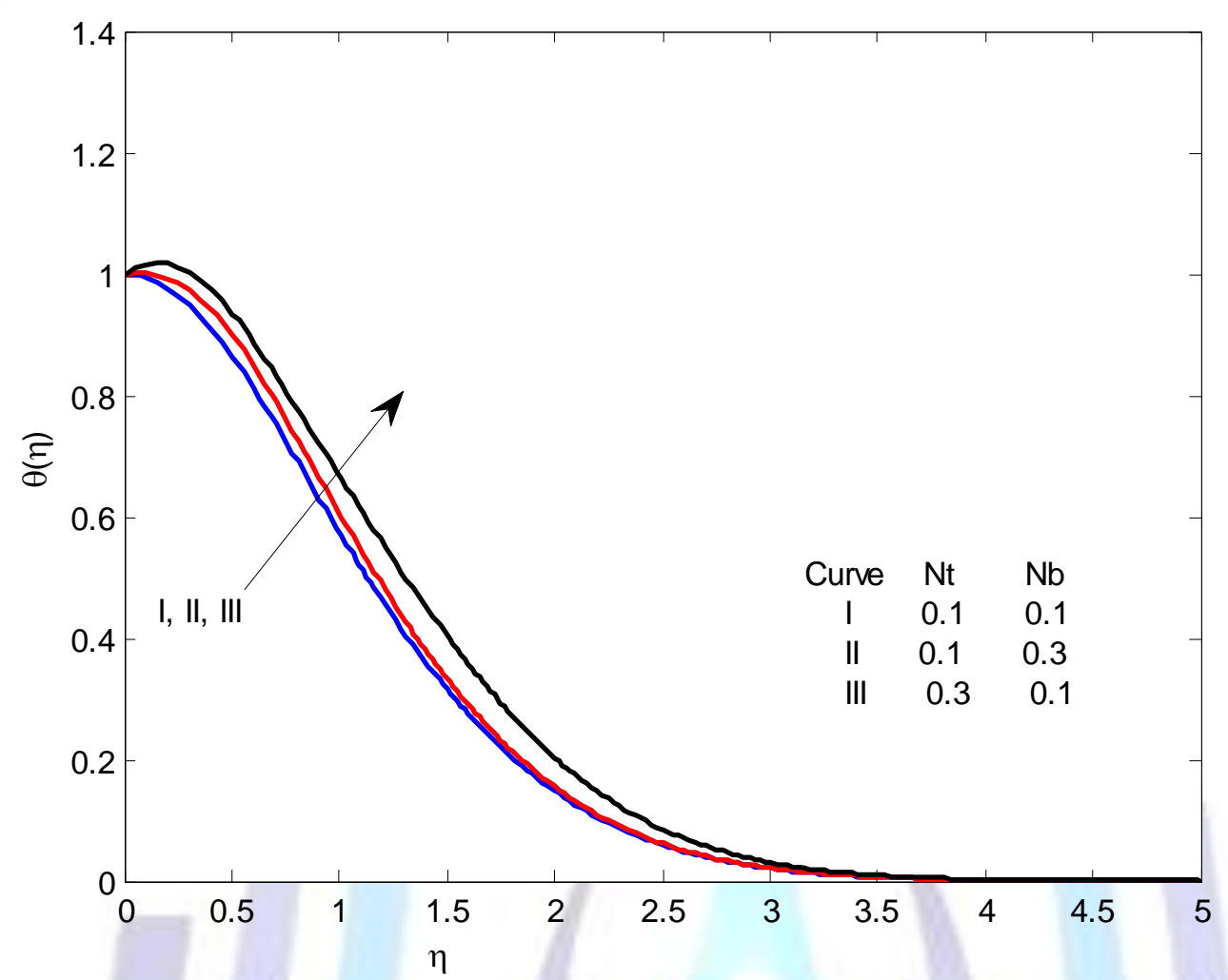

Fig.10 Effect of $\mathrm{Nt}$ and $\mathrm{Nb}$ on temperature profile 


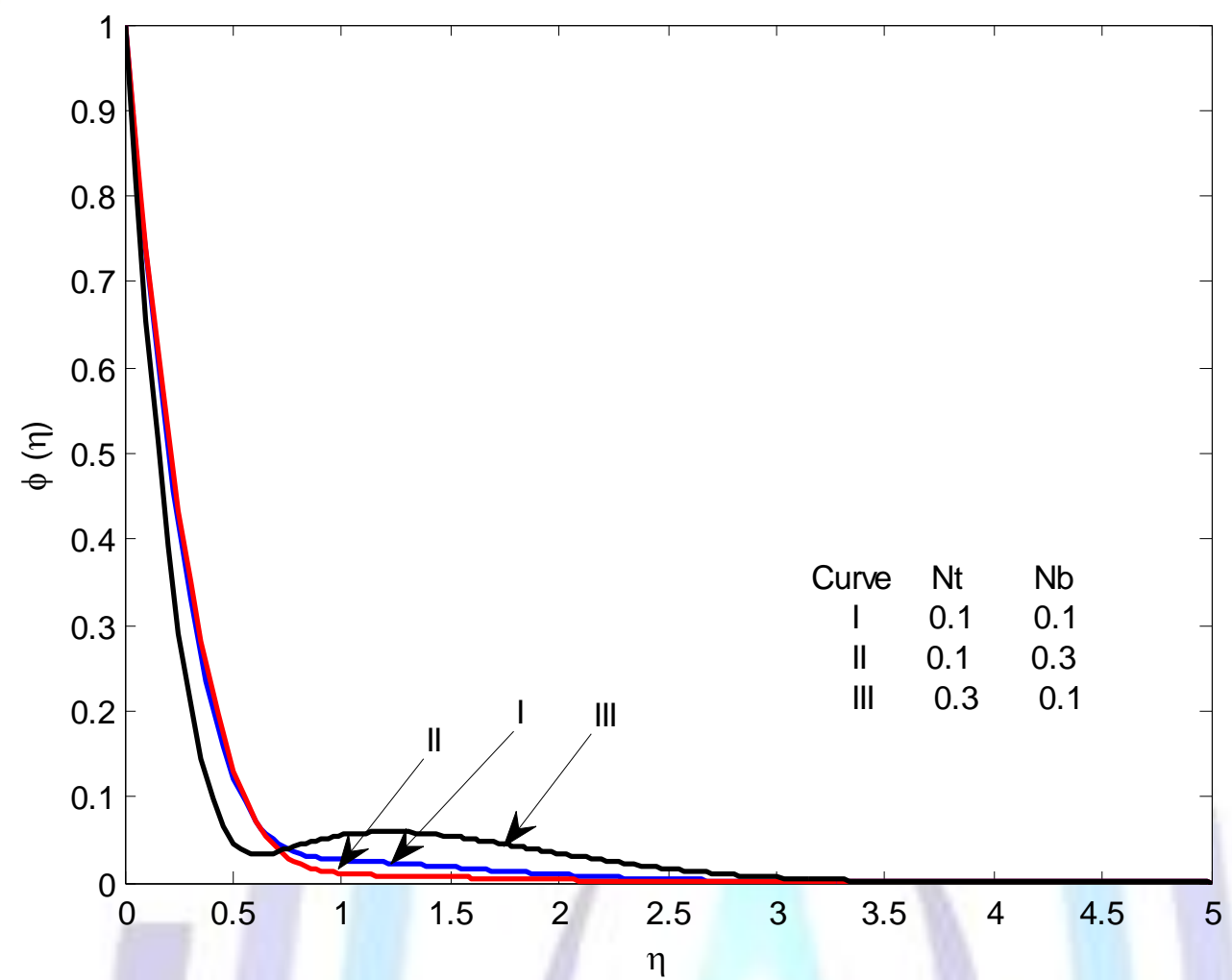

Fig. 11 Effect of $\mathrm{Nt}$ and $\mathrm{Nb}$ on concentration profile



Fig.12 Effect of $\gamma, \mathrm{Kp}$ and Du on concentration profile 